J. Clin. Chem. Clin. Biochem.

Vol. 22, 1984, pp. 319-329

\title{
Quasi-verteilungsfreie multivariate Beurteilung klinisch-chemischer Kenngrößen mit Hilfe der X-Transformation von van der Waerden
}

\author{
Von W. Rehpenning \\ Institut für Mathematik und Datenverarbeitung in der Medizin, \\ Universitäts-Krankenhaus Hamburg-Eppendorf, Hamburg, \\ K. Harm \\ Zentrallaboratorium des Allgemeinen Krankenhauses Heidberg, Hamburg,
}

J.-D. Ringe, G. H. Bützow

Kernklinik und Poliklinik des Universitäts-Krankenhauses Hamburg-Eppendorf, Hamburg und

K. D. Voigt

Abteilung für Klinische Chemie der Medizinischen Klinik, Universitäts-Krankenhaus Hamburg-Eppendorf, Hamburg

(Eingegangen am 6. Juni/2. November 1983/13. Januar 1984)

Zusammenfassung: An einem Referenzkollektiv (252 Männer, 436 Frauen) sowie bei Patientenkollektiven mit primärem Hyperparathyreoidismus (34 Männer, 67 Frauen) und mit Lebercirrhose (33 Männer) führen wir eine multivariate Datenbeurteilung durch anhand von Ergebnissen des Technicon Autoanalyzers SMA 12/60 (Natrium, Kalium, Chlorid, Gesamt-Eiweiß, anorganischer Phosphor, Harnstoff-Stickstoff, Calcium, Kreatinin, Bilirubin, Harnsäure, Albumin, Cholesterin) und des Autoanalyzers SMA 6 plus (Eisen, Kupfer, Magnesium, alkalische Phosphatase, saure Phosphatase und Glucose). Hierzu dient eine multivariate Prüfgröße, die das Alter der Personen als eine Regressorvariable enthält und eine Alterskorrektur durchführt. Die Herleitung der multivariaten Prüfgröße setzt eine multivariate Normalverteilung voraus, die im allgemeinen bei Patientenkollektiven nicht vorliegt. Wir versuchen die schiefen Verteilungen dadurch zu normalisieren, $\mathrm{da}$ wir auf die einzelnen Komponenten des jeweiligen Referenzkollektives die X-Transformation von $B$. $L$. van der Waerden ((1965) Mathematische Statistik, Springer Verlag, Berlin-Göttingen-Heidelberg) anwenden. Eingeführut wiṛ dẹ Begriff des kollektivkonformen Verhaltens. Nach unserer Definition verhält sich ein Patient in bezug auf einen Meßwert kollektivkonform zu einem Patientenkollektiv, wenn die Abweichung dieses Wertes vom geschätzten altersspezifischen Erwartungswert des Referenzkollektivs in der gleichen Richtung liegt wie die des betrachteten Patientenkollektivs. Mit diesem Vorgehen lassen sich krankheitsspezifische Abweichungsmuster errechnen, die eine deutliche Trennung der Patientenkollektive vom Referenzkollektiv und der Patientenkollektive untereinander ermöglichen. Die berechneten Abweichungsmuster geben darüberhinaus in komprimierter Form einen Einblick in pathobiochemische Veränderungen bei den untersuchten Krankheitsbildern.

Quasi-distribution-free multivariate assessment of patients' data with the aid of the $X$-transformation of van der Waerden

Summary: A reference group ( 252 males, 436 females) and a group of patients with primary hyperparathyreoidism (34 males, 67 females) and cirrhosis of the liver (33 males) were subjected to a multivariate data assessment; the analyses were performed on the Technicon autoanalyzer SMA 12/60 (sodium, potassium, 
chloride, total protein, albumin, inorganic phosphorus, cholesterol, urea nitrogen, calcium, creatinine, bilirubin, uric acid) and the SMA 6 plus (iron, copper, magnesium, alkaline phosphatase, acid phosphatase, glucose). A multivariate test statistic containing age as a regressor variable was used, thus correcting for age. Derivation of the test statistic required multivariate normality of the distributions of the clinical-chemical values, a condition which is generally not fulfilled in the data of patients. In order to arrive at the multivariate normality of the distributions, we applied the X-transformation of van der Waerden ((1965) Mathematische Statistik, Springer Verlag, Berlin-Göttingen-Heidelberg) to the marginal values. We introduced the concept of group-conformity behaviour of the patient data. According to our definition, a patient behaves in conformity with a given group of patients with respect to a clinical-chemical value, if the patients' value deviates from the estimated age-specific expected value of the reference group and the deviation lies in the same direction as that of the mean value of the respective group of patients. Using this procedure, disease-specific deviation patterns were determined from the data, thus enabling us to make clear separations of the patient groups from the reference group and from each other. Furthermore, the computed deviation patterns throw light upon the pathobiochemical modifications of the parameters in the examined diseases.

\section{Einführung}

Auf die Notwendigkeit und Nützlichkeit einer multivariaten mathematischen Beurteilung klinisch-chemischer Meßwerte ist schon oft hingewiesen worden (1-4). Nur so gelingt es, die bei Referenzpersonen vorhandenen Korrelationen zwischen den Größen bei der Beurteilung von Datensätzen angemessen zu berücksichtigen. Es gelingt mit multivariaten $\mathrm{Re}$ chentechniken einerseits, die Empfindlichkeit der Beurteilung zu steigern, andererseits, die Zahl der falsch positiven Entscheidungen drastisch zu reduzieren $(5,6)$.

Die Prüfung eines Datensatzes erfolgt durch die Berechnung multivariater Testgrößen, die im wesentlichen dem Hotelling'schen Abstandsmaß $\mathrm{T}^{2}$ entsprechen (7) und zu deren Bestimmung alle Meßwerte eines Patienten benutzt werden. Wenn, wie es oft der Fall ist, die klinischen Größen vom Alter der Person abhängen, muß die Prüfgröße noch durch ein altersabhängiges Korrekturglied normiert werden (6). Die Herleitung der Prüfgröße setzt voraus, daß sich die Daten im jeweiligen Referenzkollektiv hinreichend gut durch eine multivariate Normalverteilung beschreiben lassen. Dies schränkt naturgemäß die Anwendbarkeit der multivariaten Prüfung stark ein, weil fast alle beobachteten klinisch-chemischen Größen Abweichungen von einer Normalverteilung zeigen, sei es, daß die Verteilungen schief sind oder daß die Zusammenhänge zwischen den Größen, insbesondere auch die Altersregression, nicht linear sind. Wie weit die Anwendung der auf die Normalverteilung zugeschnittenen Methodik zulässig ist, bedarf daher jedesmal noch einer besonderen Prüfung, die jedoch ihrerseits nicht unproblematisch ist. Die Erfahrung zeigt, daß die Normalverteilungsannahmen noch am ehesten bei gesunden Referenzpersonen erfüllt sind (8). Oft gelingt es auch, durch eine einfache, meist logarithmische, Transformation eine befriedigende Approximation der Randvariablen an eine Normalverteilung $\mathrm{zu}$ erzielen.

Will man jedoch, wie es in der Anwendung besonders wünschenswert ist, das Kollektiv der gesunden Referenzpersonen nacheinander durch verschiedene Patientenkollektive ersetzen, um den Abstand des Probanden von diesem jeweiligen Kollektiv zu berechnen, so stößt die oben angedeutete Methode schnell an ihre Grenzen, weil die Abweichungen der Größen von einer Normalverteilung bei jeder Größe verschieden sind und vom Kollektiv abhängen und weil die Suche nach geeigneten Transformationen zeitraubend und unergiebig ist. Es soll daher hier die generelle Anwendung einer von van der Waerden herrührenden Transformation beschrieben und vorgeschlagen werden, die das Ziel hat, die rechnerisch einfachen, auf die Normalverteilung gegründeten Rechenverfahren auch für Patientenkollektive mit schiefen Verteilungen anwendbar zu machen.

\section{Material und Methoden}

\section{Die Daten}

Für die folgenden Untersuchungen wurden die Ergebnisse von Konzentrationsmessungen im Blutserum benutzt, die mit den TECHNICON Autoanalyzern SMA $12 / 60$ und SMA 6 plus erhalten worden waren. Die Bestimmungen erfolgten nach den im Handbuch des Herstellers angegebenen Verfahren (9): Natrium, Kalium (Flammenphotometrie), Chlorid (QuecksilberthiocyanatMethode), Gesamt-Eiweiß (Biuret-Reaktion), anorganischer Phosphor (Molybdänblau-Reaktion), Harnstoff-'Stickstoff (Diacetylmonoxim-Reaktion); Calcium (Kresolphthalein-Methode), Kreatinin (Jaffé-Reaktion), Bilirubin (Azobilirubin-Reaktion nach Jendrassik \& Gróf), Harnsäure (PhosphorwolframsäureMethode). Die Bestimmung des Albumins erfolgte mit der Bromkresolgrün-Methode, die des Cholesterins mit der Cholesterinoxidase-Phenol-Aminophenazon-Methode. Eisen wurde mit Batho- 
phenanthrolinsulfonat, Kupfer mit Oxalyl-dihydrazid und Magnesium mit Xylidylblau bestimmt. Die Bestimmung der alkalischen und sauren Phosphatase erfolgte kontinuierlich, und zwar mit den Substraten $p$-Nitrophenylphosphat bei einem pH-Wert von 10,25 bei $37,5^{\circ} \mathrm{C}$ bzw. durch Hydrolyse von Phenylphosphat bei einem $\mathrm{pH}$-Wert von 4,8 bei $37^{\circ} \mathrm{C}$. Außerdem standen noch die Werte der Enzyme Aspartataminotransferase, Alaninaminotransferase und $\gamma$-Glutamyltranspeptidase zur Verfügung, die mit dem $\mathrm{Ne}$ theler + Hinz-Enzymautomaten 5010 mit der optimierten Standardmethode der Deutschen Gesellschaft für Klinische Chemie bestimmt wurden.

Für die Anwendung der mathematischen Theorie wurden die Datensätze von einem Referenzkollektiv und zwei Patientenkollektiven benutzt. Referenzkollektive waren 252 Männer und 436 Frauen, die im Verlauf der Jahre 1979 und 1980 einer routinemäBigen Untersuchung unterzogen und als gesund eingestuft worden waren. Bei den Patienten handelte es sich um 34 Männer und 67 Frauen mit primärem Hyperparathyreoidismus und 33 Männer mit Lebercirrhosen. Die Diagnose bei den Personen mit Hyperparathyreoidismus stützte sich auf anamnestische und klinische Befunde, Röntgenuntersuchungen und Knochenhistologie und wurde bei allen 101 Fällen operativ bestätigt. Bei den Männern mit Lebercirrhosen war die Diagnose bioptisch gesichert. In den meisten Fällen lag eine alkoholische, in einigen Fällen eine posthepatische Cirrhose vor. Bei allen Patienten waren die hier benutzten Laborwerte vor der Behandlung gemessen worden.

\section{Die multivariate Prüfung}

Für die Ableitung der multivariaten Prüfgröße wird zunächst angenommen, daß sich die Daten des Referenzkollektives durch eine multivariate Normalverteilung beschreiben lassen. Das Alter der Personen sei mit $x_{0}$, die Meßwerte der klinisch-chemischen Kenngrößen mit $x_{1}, \ldots, x_{p}$ bezeichnet. Diese Werte seien zu einem Vektor $\dot{\mathfrak{c}}$ mit $\dot{\mathrm{q}}=\left(\mathrm{x}_{0}, \mathrm{x}_{1}, \ldots, \mathrm{x}_{\mathrm{p}}\right)^{\prime}$ zusammengefaßt. Der Vektor der Erwartungswerte $\mu_{i}=E\left(x_{i}\right)(i=0, \ldots, p)$ sei mit $\dot{\mu}$ bezeichnet. Die Transposition eines Vektors sei hier und im folgenden durch ein Apostroph angedeutet. Die gemeinsame Wahrscheinlichkeitsdichte aller Größen ergibt sich nach diesen Voraussetzungen zu:

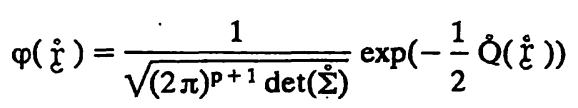

Hierbei ist $Q ̊(\mathfrak{x})$ die quadratische Form:

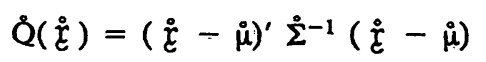

und $\stackrel{\circ}{\Sigma}$ die Matrix der Kovarianzen:

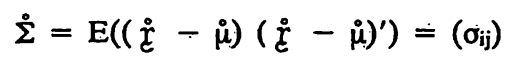

Weil die Kovarianzmatrix symmetrisch ist, gilt für alle $i j: \sigma_{i j}=\sigma_{j i}$. Die Determinantenfunktion wird mit det bezeichnet. Die Varianzen $\sigma_{i i}$ und die Kovarianzen $\sigma_{i j}$ hängeng natürlich vom jeweils zugrunde gelegten Referenzkollektiv ab. Wenn man den Datenvektor eines Probanden auf Abweichung vom Referenzkollektiv beurteilen will, so muß man zunächst den Einflu'B des Alters $x_{0}$ herausrechnen. Hierzu muß man die bedingte Verteilung der Werte bei festgehaltenem Alter betrachten. Die bedingte Wahrscheinlichkeitsdichte läßt sich erhalten, wenn man die Kovarianzmatrix $\stackrel{\circ}{\Sigma}$ geeignet zerlegt:

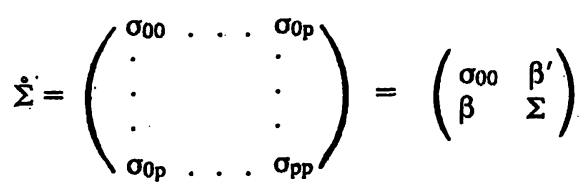

Dabei ist $\beta=\left(\sigma_{01}, \ldots, \sigma_{0 p}\right)^{\prime}$ der Vektor der Kovarianzen zwischen dem Alter $x_{0}$ und den klinisch-chemischen Kenngrößen. $\Sigma$ ist die
Matrix der Kovarianzen der Größen untereinander ohne Berücksichtigung des Alters. Für die bedingte Wahrscheinlichkeitsdichte ergibt sich danach:

$$
\varphi\left(x_{1}, \ldots, x_{p} \mid x_{0}\right)=\frac{1}{\sqrt{(2 \pi)^{p} \operatorname{det}(\Gamma)}} \exp \left(-\frac{1}{2} Q(\mathfrak{x})\right)
$$

mit $\mathfrak{E}=\left(\dot{x}_{1}, \ldots, x_{p}\right)^{\prime}$ und der quadratischen Form:

$$
Q(\mathfrak{E})=(\mathfrak{x}-\mathfrak{y})^{\prime} \Gamma^{-1}(\mathfrak{z}-\mathfrak{y})
$$

Dabei ist die bedingte Kovarianzmatrix $\Gamma$ gegeben durch:

$$
\Gamma=\Sigma-\beta \beta^{\prime} / \sigma_{00}
$$

$\mathfrak{y}$ 'ist der Regressionsvektor:

$$
\mathfrak{l}=\mu+\left(x_{0}-\mu_{0}\right) \beta / \sigma_{00}
$$

$\mathfrak{x}$ und $\mathfrak{y}$ sind also $p$-dimensionale Vektoren. Die quadratische Form (Gl. 5) stellt nun das gesuchte Abstandsmaß dar. Bei bekannten Erwartungswerten und Kovarianzen wäre die Form $\chi^{2}$ verteilt mit $p$ Freiheitsgraden. In der Anwendung müssen die unbekannten Größen durch ihre Stichprobenschätzungen ersetzt werden. Es ergibt sich dann die empirische vektorielle Regressionsgleichung mit dem Alter $x_{0}$ als Regressorvariablen:

$$
\hat{\mathfrak{y}}=\overline{\mathfrak{z}}+b\left(x_{0}-\bar{x}_{0}\right)
$$

Für die Elemente $b_{0 i}$ des Vektors $b$ der empirischen Regressionskoeffizienten gelten dann die Beziehungen $(i=1, \ldots, p)$ :

$$
b_{0 i}=\frac{\sum_{j=1}^{n}\left(x_{0}^{(j)}-\bar{x}_{0}\right) x_{i}^{(j)}}{\sum_{j=1}^{n}\left(x_{0}^{(j)}-\bar{x}_{0}\right)^{2}}
$$

Hierbei ist $\mathbf{n}$ der Stichprobenumfang des Referenzkollektivs; der Index $j$ bezieht sich auf die verschiedenen Referenzpersonen. Insgesamt ergibt sich, da $B$ sich die Regressionsgleichungen schreiben lassen:

$$
\hat{\mathfrak{y}}=\sum_{j=1}^{n}\left(\frac{1}{n}+c_{j}\right) \mathfrak{x}^{(j)}
$$

Hierbei sind die $c_{j}$ feste Zahlen, für die gilt: $\Sigma c_{j}=0$. Bezeichnet VAR den Kovarianzoperator, so ergibt sich aus (GI. 10) unter Berücksichtigung der Unabhängigkeit der Stichprobenvektoren $\mathfrak{E}^{(j)}$ nach leichten $Z$ wischenrechnungen für die Kovarianzmatrix der Regression $\mathfrak{h}$ :

$$
\operatorname{VAR}(\hat{\mathfrak{y}})=\left[\frac{1}{n}+\frac{\left(x_{0}-\bar{x}_{0}\right)^{2}}{(n-1) s_{00}}\right] \operatorname{VAR}(\mathfrak{E})
$$

Hierbei bezeichnet $s_{00}$ die empirische Varianz der Werte von $x_{0}$ und $\operatorname{VAR}(\mathfrak{E})$ die Kovarianzmatrix des Vektors $\mathfrak{x}$ bei festen Werten von $x_{0}$, also die Kovarianz um die Regressionsgerade. Diese ist durch (Gl. 6) gegeben. Ihre Schätzung sei mit $G$ bezeichnet. Sind $x_{0}, \mathfrak{x}$ die Meßwerte eines vom Referenzkollektiv unabhängigen Probanden, so ergibt sich schließlich aus den obigen Gleichungen für die Abweichung $\mathfrak{t}-\hat{\mathfrak{h}}$ als Kovarianzmatrix:

mit:

$$
\operatorname{VAR}(\mathfrak{x}-\hat{\mathfrak{y}})=B_{n}^{2} \operatorname{VAR}(\mathfrak{x})=B_{n}^{2} G
$$

$$
\mathrm{B}_{\mathrm{n}}^{2}=\frac{\mathrm{n}+1}{\mathrm{n}}+\left(\mathrm{x}_{0}-\overline{\mathrm{x}}_{0}\right)^{2} /\left((\mathrm{n}-1) \mathrm{s}_{00}\right)
$$

Es folgt, daß das quadratische Abstandsmaß $V^{2}$ mit:

$$
V^{2}=(\mathfrak{E}-\hat{\mathfrak{y}})^{\prime} G^{-1}(\mathfrak{x}-\hat{\mathfrak{y}}) / B_{n}^{2}
$$

Hotelling-verteilt ist, d.h. daß die Größe:

$$
F=(n-p) V^{2} /((n-1) p)
$$


F-verteilt ist mit $\mathbf{p}$ und $n-p$ Freiheitsgraden. Hält man das Referenzkollektiv fest, so ergibt sich für $V^{2}$ eine nichtzentrale $\chi^{2}$-Verteilung mit $\mathrm{p}$ Freiheitsgraden und einem Nichtzentralitätsparameter, der umso kleiner ist, je näher die Schätzungen der Kovarianzen und Erwartungswerte bei den wahren Werten liegen. Für hinreichend große $n$ kann man daher auch eine $\chi^{2}$-Verteilung mit $p$ Freiheitsgraden als Prüfverteilung nehmen, wie wir es im folgendem tun wollen (siehe auch Rehpenning (6)). Betrachtet man daher alle Datensätze von Probanden als nicht pathologisch, bei denen die hieraus berechnete Prüfgröße $V^{2}$ ein Fraktil der Prüfverteilung nicht überschreitet, so ergibt sich als Toleranzbereich (mehrdimensionales Prognosegebiet) ein Schlauch um die Regressionsgerade (Gl. 8), der von einem Hyperboloid berandet ist und der an den Enden (bei niedrigem und hohem Alter $\mathrm{x}_{0}$ ) weiter geöffnet ist als in der Mitte. Diese Situation ist in der Abbildung 1 graphisch am Beispiel zweier abhängiger Größen veranschaulicht.

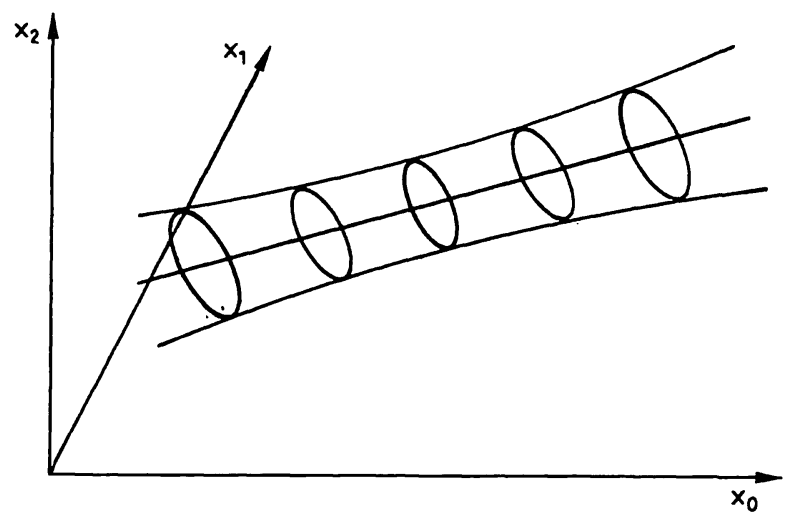

Abb. 1. Hyperbolischer Referenzbereich am Beispiel zweier klinisch-chemischer Kenngrößen mit der Regressorvariablen $\mathrm{x}_{0}$. Man erkennt den elliptischen Querschnitt des Referenzbereiches. Die Form der Ellipse ist durch die Korrelation zwischen $x_{1}$ und $x_{2}$ bestimmt. Die Regressionsgerade geht durch die Mittelpunkte der Ellipsen. Einzelheiten siehe Text.

\section{Die X-Transformation}

Die Ableitung der multivariaten Prüfgröße setzte multivariat normalverteilte Daten voraus. Das bedeutet, daß eigentlich nur solche Kollektive als Referenzkollektive für eine Datenprüfung herangezogen werden dürfen, deren Verteilung multivariat normal ist. Weil nun bei allen realen Kollektiven, insbesondere bei $\mathrm{Pa}$ tientenkollektiven, unter Umständen sehr große Abweichungen von einer Normalverteilung zu beobachten sind, kann man die Prüfgröße (Gl. 14) nur als mehr oder weniger grobe Approximation ansehen für die Prüfung der Datensätze. Man kann die Prüfung jedoch wesentlich verbessern, wenn es gelingt, die Datenvektoren so zu transformieren, daß in den Daten des Referenzkollektivs in den Randverteilungen Normalität hergestellt wird. Um die aufwendige Suche nach geeigneten Transformationen für die einzelnen Variablen zu umgehen, soll hier durchweg die Anwendung der X-Transformation von van der Waerden (10) vorgeschlagen und praktiziert werden, die so definiert ist: Zunächst werden die Meßwerte $x_{i}^{(j)}$ auf die übliche Weise in Rangzahlen $R_{i}^{(j)}$ verwan-
delt:

$$
x_{i}^{(j)} \rightarrow R_{i}^{(j)}(i=1, \ldots, p ; j=1, \ldots, n)
$$

Die Ränge werden dann mit der Formel: $Y=R /(n+1)$ in das offene Intervall $(0,1)$ abgebildet. Bedeutet $\Phi$ das Integral der Standardnormalverteilung, so wird schließlich gesetzt:

$$
X_{i}^{(j)}=\Phi^{-1}\left(Y_{i}^{(j)}\right)
$$

Die so berechneten Zahlen $X_{i}^{(j)}$ sollen „X-Ränge der $j$-ten Person zur i-ten. klinisch-chemischen Kenngröße" heißen. Die durch
(GI. 15) definierte Abbildung ist eine diskretisierende Abbildung mit endlichem, im wesentlichen (bei Abwesenheit von Bindungen sogar nur) vom Stichprobenumfang $n$ abhängenden Wertebereich. Die empirische Verteilungsfunktion der X-Ränge liegt jedoch, bis auf den Faktor $(n+1) / n$, genau auf der Verteilungsfunktion der standardisierten Normalverteilung, und darin liegt der Wert der X-Transformation und der Grụnd für ihre Anwendung.

Die X-Transformation soll also im folgenden komponentenweise auf die Daten des jeweiligen Referenzkollektivs angewandt werden. Der Datensatz eines mit dem Referenzkollektiv zu vergleichenden Probanden soll folgendermaßen transformiert werden: Für jede Komponente wird geprüft, welchem untransformierten Wert des Referenzkollektivs diese Komponente am nächsten liegt, daraufhin wird ihr dann der entsprechende $X$-Wert zugeordnet. Die Probandenwerte werden also, anders ausgedrückt, in das mehrdimeñsionale X-Raster „eingeschätzt". Die mit den so erhaltenen transformierten Probandenwerten berechnete Prüfgröße nach (Gl.14) soll mit $V_{X}^{2}$ bezeichnet werden. Hierbei treten also die X-Ränge an die Stelle der ursprüunglichen Meßwerte, natürlich auch im Referenzkollektiv. Der Index , $X$ “ soll daran erinnern, da $B$ die Meßwerte transformiert wurden.

Wie bei allen Rangtransformationen, so entsteht auch bei den $X$ transformierten Werten eine leichte negative Korrelation zwischen den $X$-Rängen der verschiedenen Referenzpersonen und $z w a r$ ist für alle $i, j, k$ mit $j \neq k: r\left(X_{i}^{(j)}, X_{i}^{(k)}\right)=-1 /(n-1)$. (Man vergleiche hierzu die Ausführungen bei van der Waerden (10)). Der Grund hierfür liegt, etwas ungenau ausgedrückt, darin, daß nach der Einordnung von $\mathbf{n}-1$ Werten der Rangplatz des letzten Wertes festliegt. Diese Korrelation ist nicht nur sehr klein, sondern würde auch nur dann bemerkbar werden, wennn immer wieder Stichproben vom Umfang n gezogen und dann die X-Ränge berechnet würden. $\mathrm{Da}$ es hier aber nur darauf ankommt, für das Referenzkollektiv geeignete transformierte Werte zu bekommen, diese dạnn aber festgehalten werden, spielen solche Korrelationen bei den jetzigen Uberlegungen gar keine Rolle.

Weil theoretische Aussagen über die exakte Verteilung der diskreten Prüfgröße $V_{X}^{2}$ kaum zu bekommen sind, sollen empirische Dichteschätzungen mit Hilfe normalverteilter Kernschätzer (11) vorgenommen und diese mit der Dichte der $\chi^{2}$-Verteilung verglichen werden. Dies soll für verschiedeǹe Kollekktive, die einmal als Referenz-, dann als Vergleichskollektive dienen, graphisch dargestellt werden.

\section{Kollektivkonformes Verhalten}

$\mathrm{Da}$ die multivariate Prüfgröße gewișsermaßen ein $\mathrm{Maß}$ für den Abstand eines Probanden vom Zentrum èines Referenzkollektivs darstellt, bedeutet eine Signifikanz der Prüfgröße, daß der Proband in seinen Werten aller Wahrscheinlichkeit nach nicht in das betreffende Referenzkollektiv paßt, also einem anderen Kollektiv angehört. Hieraus ist noch nicht ersichtlich, in welchen klinischchemischen Kenngrößen sich der Proband wesentlich vom Referenzkollektiv abhebt. Daher ist es zweckmäßig, Untermengen des gegebenen Kenngrößensatzes zu betrachten. Entstammt der Proband einem Kollektiv mit einer fest definierten Krankheit, so ist es weiterhin nützlich, Gemeinsamkeiten bei den verschiedenen Personen des Patientenkollektivs herauszuarbeiten. Hierzu kann das Konzept des ,,kollektivkonformen Verhaltens“ beitragen. Wir wollen sagen, ein Patient verhalte sich in bezug auf eine klinischchemische Größe kollektivkonform, wenn die Abweichung dieser Größe vom geschätzten altersspezifischen Erwartungswert in der gleichen Richtung liegt wie die des Mittelwertes des Patientenkollektivs. Um solche kollektivkonformen Verhaltensweisen nicht nur für die einzelnen Größen, sondern auch für Größenkombinationen zu finden, wurde folgendes Verfahren angewandt: Es wurden alle möglichen Kombinationen von je vier aus den p Kenngrößen gebildet und für alle Personen berechnet, für welche Viererkombination die multivariate Prüfgröße ihren größten Wert annimmt. Dann wurde abgezählt, wỉe oft alle möglichen 
Kenngrößenpaare in diesen individuellen Maximalkombinationen vertreten waren. Die Ergebnisse dieser Rechnungen wurden in graphischer Form dargestellt. Um den Einfluß der X-Transformation abschätzen zu können, wurden für den Vergleich der Patientenkollektive mit den gesunden Referenzkollektiven die Rechnungen sowohl mit den Originaldaten als auch mit den X-transformierten Werten durchgeführt und die Ergebnisse miteinander verglichen.

\section{Ergebnisse}

\section{Die X-Transformation}

Soweit stichprobenartig durchgeführte Inspektion der zweidimensionalen Randverteilungen eine Beurteilung der angestrebten Normalität der transformierten Werte gestattete, führt die X-Transformation bei den gesunden Referenzpersonen zu einer hervorragenden Normalisierung der Daten. Als Beispiel zeigen die Abbildungen 2 und 3 den Zusammenhang zwischen der sauren Phosphatase und der Alaninaminotransferase bei 171 Männern sowohl bei den Originalwerten als auch bei den X-transformierten Werten. Wie man sieht, weist die Alaninaminotransferase eine merkliche positive Schiefe auf, die nach der Transformation völlig verschwindet. Bei allen durchgerechneten Beispielen war eine Abweichung von der Linearität des Zusammenhangs nicht zu erkennen. Es zeigt sich überdies, daß die Anwendung der X-Transformation auch bei den $\mathrm{Pa}$ tientenkollektiven zu sehr befriedigenden Verteilungen führt. Ein Beispiel zeigt die Abbildung 4. Hier ist der Zusammenhang zwischen dem Albumin und dem Calcium in den transformierten Werten bei zwei Patientenkollektiven dargestellt. Einmal wurden die Männer mit Lebercirrhosen, zum zweiten die Männer mit Hyperparathyreoidismus als Referenzkollektive gewählt. Die Referenzpersonen sind jeweils durch Kreise dargestellt. Dickere Kreise bedeuten, daß mehrere Werte auf dieselbe Stelle fallen (infolge von Bindungen in den gerundeten Daten). Die Werte des in das endliche X-Raster eingeschätzten Vergleichskollektives sind durch Kreuze angezeigt. Hierbei ist wieder durch die Stärke der Symbole angedeutet, wieviele Patienten auf dieselbe Position fallen. Infolge der großen Unterschiede zwischen den beiden Kollektiven in den Calciumwerten liegen die Punkte des Vergleichskollektivs jeweils auf dem Rand des X-Gitters. Die Information, wie weit die Werte von dieșem Rand entfernt liegen, geht verloren. Dies ist aber bei allen verteilungsfreien Methoden unvermeidlich.

Immerhin wird deutlich, daß die Werte bei den beiden jeweiligen Referenzkollektiven recht gut durch zweidimensionale Normalverteilungen beschrieben werden können. Während bei den Lebercirrhotikern eine sehr hohe Korrelation zwischen dem Albumin

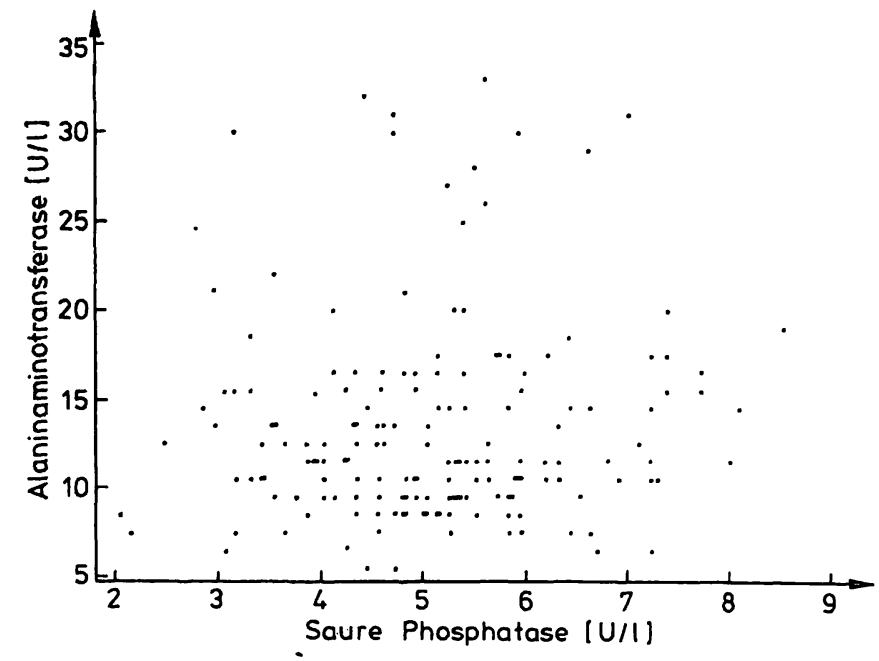

Abb. 2. Der Zusammenhang zwischen der sauren Phosphatase und der Alaninaminotransferase bei Referenzpersonen $\left(n=171 \delta^{\prime}\right)$. Man erkennt die positive Schiefe der Alaninaminotransferase-Werte. Der Pearsonsche Korrelationskoeffizient ist $r=0,107$.

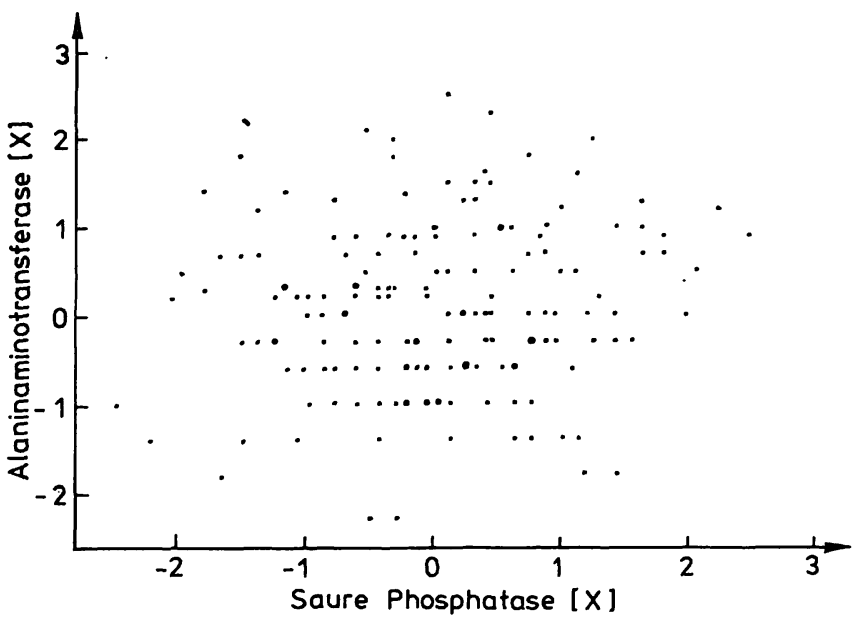

Abb. 3. Der Zusammenhang zwischen der sauren Phosphatase und der Alaninaminotransferase bei Referenzpersonen $(\mathrm{n}=171 \delta)$ nach der Anwendung der X-Transformation. Die Schiefe der Alaninaminotransferase-Werte ist verschwunden. Der Pearsonsche Korrelationskoeffizient ist $\mathrm{r}=0,128$.

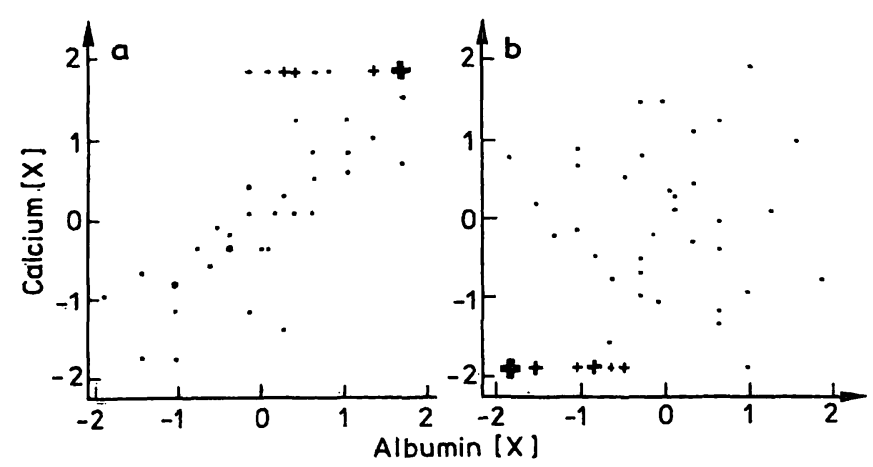

Abb. 4. Die Auswirkung der X-Transformation auf Patientendaten. Die kleinen Kreise kennzeichnen die Werte des jeweiligen Referenzkollektivs

a) Lebercirrhose

b) Hyperparathyreoidismus,

die Kreuze die Werte des damit zu vergleichenden Kollektivs. Bei den Patienten mit Lebercirrhose ergibt sich der Pearsonsche Korrelationskoeffizient als $r=0,79$, bei den Patienten mit Hyperparathyreoidismus als $r=$ $-0,12$. Einzelheiten siehe Text. 
und dem Calcium besteht, ist diese Korrelation bei den Patienten mit Hyperparathyreoidismus praktisch verschwunden. Betrachtet man andere zweidimensionale Randverteilungen, so ergeben sich ebenfalls befriedigende Darstellungen nach der Durchführung der $\mathrm{X}$-Transformation.

\section{Die Verteilung der Prüfgrößen}

Um die Verteilung der Prüfgröße $V_{X}^{2}$ empirisch zu überprüfen, wurden die $V_{X}^{2}$-Werte für die gesunden Männer und Frauen berechnet und eine formale Dichteschätzung mit Hilfe normalverteilter Kerne durchgeführt, die der Darstellung durch Histogramme überlegen ist. Den Vergleich der Schätzung mit der $\chi^{2}$-Verteilung erlaubt die Abbildung 5. Man erkennt, daß die empirische Dichteschätzung durch die $\chi^{2}$-Verteilung exzellent beschrieben werden kann. Lediglich bei höheren $\mathrm{V}_{\mathrm{X}}^{2}$-Werten sind kleine $\mathrm{Ne}$ benmaxima angedeutet, die vielleicht am besten durch noch verbleibende Inhomogenitäten in den Referenzkollektiven zu erklären sind. Insgesamt fällt dieser formale Vergleich zur Prüfung der Transfor- mationsmethode auf ihre Brauchbarkeit positiv aus.

Ersetzt man die Referenzkollektive der Gesunden durch die Patientenkollektive, so ergeben sich Verteilungen, die in der Abbildung 6 dargestellt sind. Auch hier läßt sich sagen, daß die $\chi^{2}$-Verteilung die Prüfverteilungen im ganzen recht befriedigend darstellt. Man muß allerdings bei diesen Kollektiven den relativ geringen Stichprobenumfang berücksichtigen.

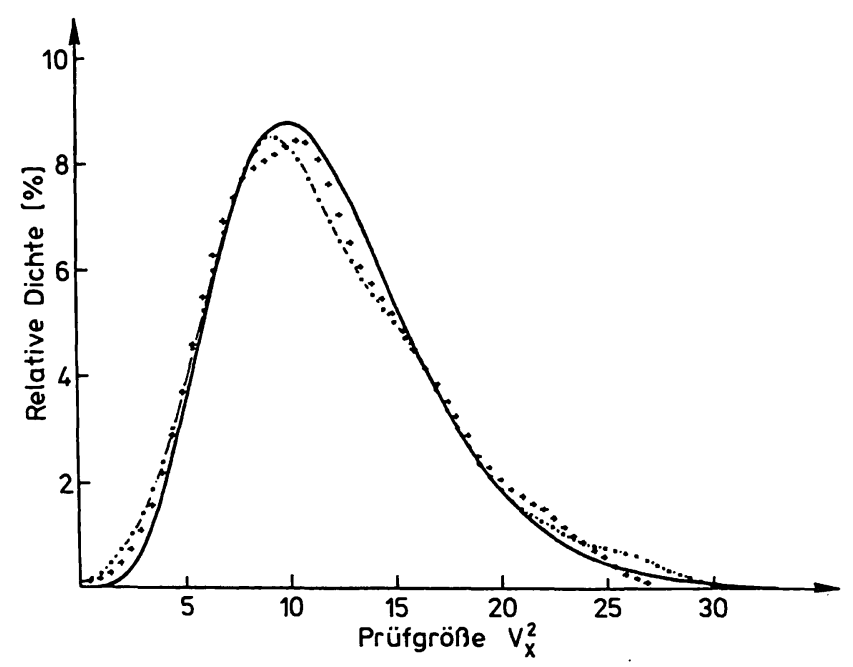

Abb. 5. Der Vergleich der empirischen Verteilung der Prüfgröße $\mathrm{V}_{\mathrm{X}}^{2}$ mit der theoretisch erwarteten $\chi^{2}$-Verteilung mit zwölf Freiheitsgraden für die Daten des SMA 12/60. Man erkennt die ausgezeichnete Ubereinstimmung der empirischen Dichteschätzung mit der Dichte der $\chi^{2}$-Verteilung. Die strichpunktierte Linie kennzeichnet die Dichteschätzung bei 436 Frauen, die Kreuze die bei 252 Männern. Die ausgezogene Linie stellt die Dichte der $\chi^{2}-$ Verteilung dar. Einzelheiten siehe Text.
Der praktische Wert der Prüfgrößen liegt indessen darin, daß sich mit ihrer Hilfe die Kollektive trennen lassen. In der Abbildung 7 ist die Verteilung der Prüfgröße $V_{X}^{2}$ für die Patientenkollektive berechnet, wenn man diese mit den geschlechtsspezifischen Referenzkollektiven der Gesunden vérgleicht. Man erkennt, daß fast alle Werte in den kritischen Bereich $\left(V_{X}^{2}>21,0\right)$ fallen, also als pathologisch anzusehen sind. Gleichzeitig wird jedoch ebenfalls deutlich, daß eine Diskriminierung zwischen den Patientenkollektiven so noch nicht möglich ist. Dies wird aber anders, wenn man das Referenzkollektiv der Gesunden dưrch ein Patientenkollektiv ersetzt und die entsprechende Prüfgröße in bezug auf dieses Kollektiv berechnet. Ein Beispiel sieht man in der Abbildung 8, in der die Verteilung der Prüfgröße bèi Männern mit Hyperparathyreoidismus, bezogen auf die Männer mit Lebercirrhosen, dargestellt ist. Man erkennt, daß nunmehr die Kollektive weitgehend getrennt erscheinen. Ganz entsprechende Bilder ergeben sich auch bei anderen Kombinationen von Kollektiven. Noch verbleibende Überschneidungen lassen sich weitgehend auf die geringen Stichprobenumfänge zurückführen, die ja den Wertebereich der X-Ränge einschränken.

\section{Kollektivkonformes Verhalten}

Zur Darstellung gemeinsamer Abweichungen der Patientenkollektive von den Referenzkollektiven wurden graphische Strukturen gewählt. Hierbei wurden als Symbole für die betrachtèteñ klinisch-chemi-

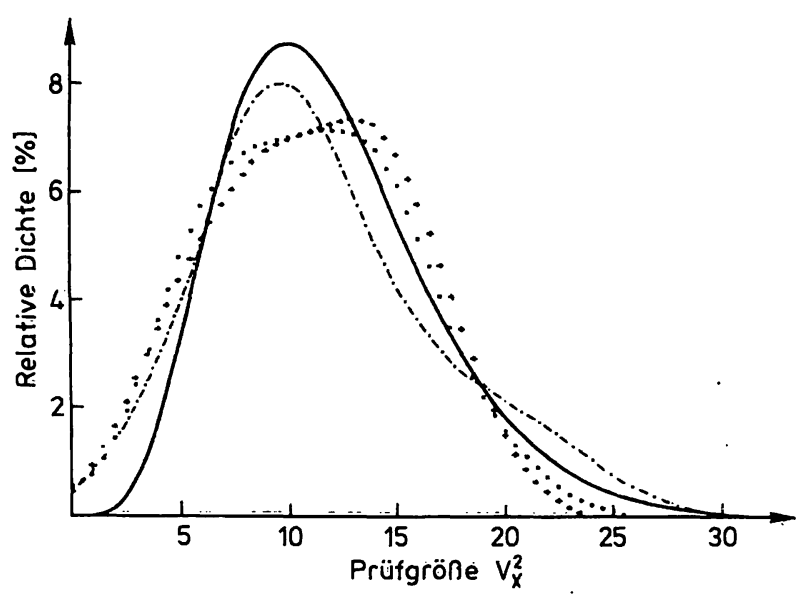

Abb. 6. Der Vergleich der empirischen Verteilungen der Prüfgröße $V_{x}^{2}$ mit der $\chi^{2}$-Verteilung für die Kenngrößen des SMA 12/60. Referenzkollektive sind diesmal die Patientenkollektive. Die strichpunktierte Linie bezieht sich auf 67 Frauen mit Hyperparathyreoidismus, die punktierte Linie auf 34 Männer mit Hyperparathyreoidismus und die Kreuze auf 33 Männer mit Leberrcirrhose. Die ausgezogene Linie stellt die Dichte der $\chi_{i}^{2}$ Verteilung mit zwölf Freiheitsgraden dar. Einzelheiteñ siehe Text. 


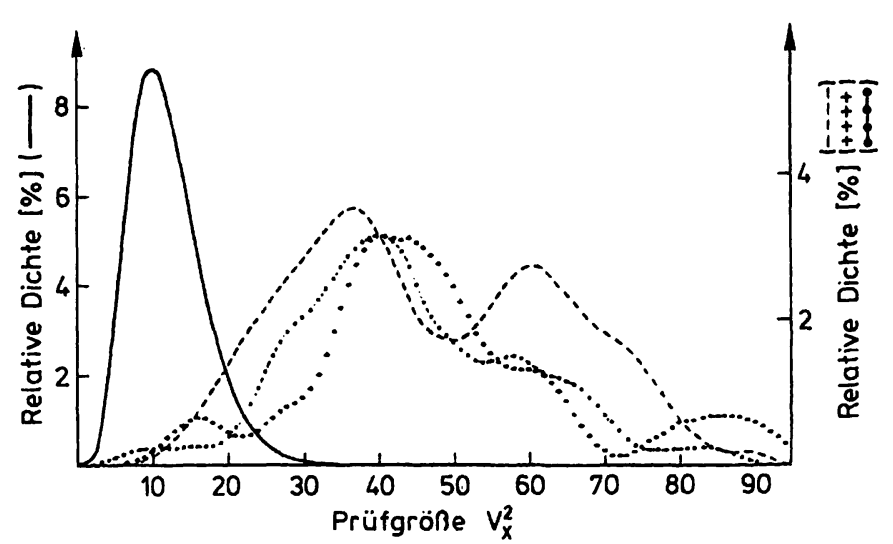

Abb. 7. Der Vergleich der empirischen Verteilungen der Prüfgröße $V_{x}^{2}$ mit der $\chi^{2}$-Verteilung. Bezugskollektive sind die Referenzpersonen, Prüfkollektive sind die Patientenkollektive. Die gestrichelte Linie bezieht sich auf 67 Frauen mit Hyperparathyreoidismus, die strichpunktierte Linie auf 34 Männer mit Hyperparathyreoidismus, die Kreuze auf 33 Patienten mit Lebercirrhose. Man erkennt, daß die Werte der Prüfgröße bei den Patienten deutlich nach oben in signifikante Bereiche verschoben sind. Einzelheiten siehe Text.

schen Kenngrößen kleine Kreise auf einem großen Kreis angeordnet. Durch eingezeichnete Plus- und Minuszeichen wird angedeutet, in welcher Richtung sich die Patienten im Mittel von den Referenzkollektiven unterscheiden. Die Stärke der Abweichungen soll durch die Dicke der Symbole qualitativ angezeigt werden. Zusätzlich wurde für jeden Patienten berechnet, für welche vier aus den zwölf KenngröBen des SMA 12/60 die zugehörige Prüfgröße ihren maximalen Wert annimmt (individuelle Maximalkombination). Dem Konzept vom kollektivkonformen Verhalten folgend, wurde abgezählt, wie oft einzelne Kenngrößenpaare in den individuellen Maximalkombinationen vorkommen. Diese Häufigkeiten wurden, von einer gewissen Schwelle ab, durch Querverbindungen zwischen den Symbolen qualitativ gekennžeichnet. Die so entstandene Graphik wurde „diagnostische Wertigkeit" bżw. „Abweichungsmuster" der klinisch-chemischen KenngröBen für das jeweilige Patientenkollektiv genannt. Um den Einfluß der X-Transformation deutlich zu machen, wurden die Rechnungen einmal mit den untränsformierten Werten (d.h. weder die Referenznoch die Patientenwerte wurden transformiert) als auch mit den transformierten Werten durchgeführt und die Ergebnisse einander gegenübergestellt.

Die Abbildung 9 zeigt das berechnete Abweichungsmuster mit den Originaldaten, die Abbildung 10 das entsprechende Muster für die X-Ränge bei den Männern mit Lebercirrhosen. Beide Bilder zeigen viele Gemeinsamkeiten. Beherrscht werden sie durch eine starke Abnạme des Albumins und durch

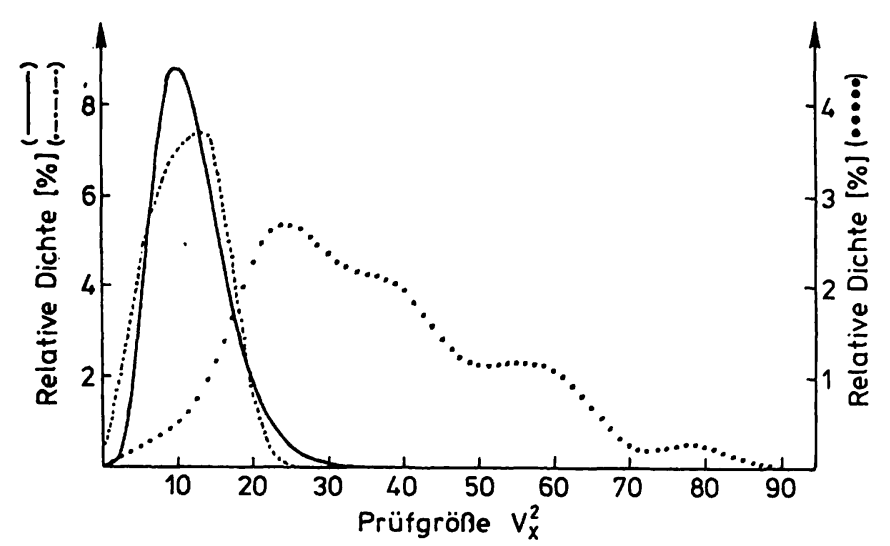

Abb. 8. Die Trennung zweier Patientenkollektive mit der Prüfgröße $V_{x}^{2}$ für die Kenngrößen des SMA 12/60. Referenzkollektiv bilden die Männer mit Lebercirrhosen. Uberscheidungen sind auf die geringe Stichprobengröße des Referenzkollektivs zurückzuführen. Dic strichpunktierte Linie kennzeichnet die Dichteschätzung bei Patienten mit Lebercirrhosen (Referenzkollektiv), die punktierte Linie stellt die Dichteschätzung bei 34 Männern mit Hyperparathyreoidismus dar. Einzelheiten siehe Text.

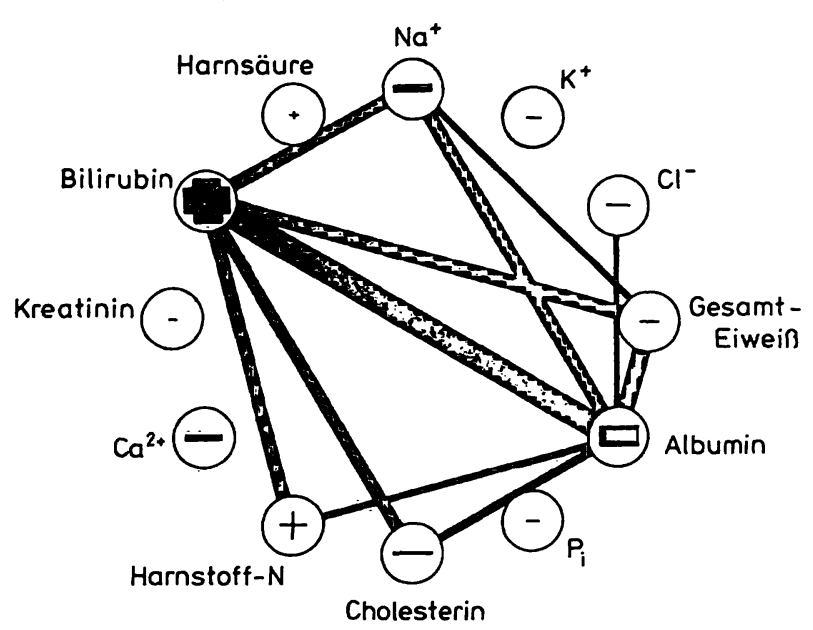

Abb. 9. Krankheitsspezifisches Abweichungsmuster der Patienten mit Lebercirrhosen $(n=33 \delta)$, bezogen auf das $R e-$ ferenzkollektiv der gesunden Männer. Die Werte wurden nicht transformiert. Einzelheiten siehe Text.

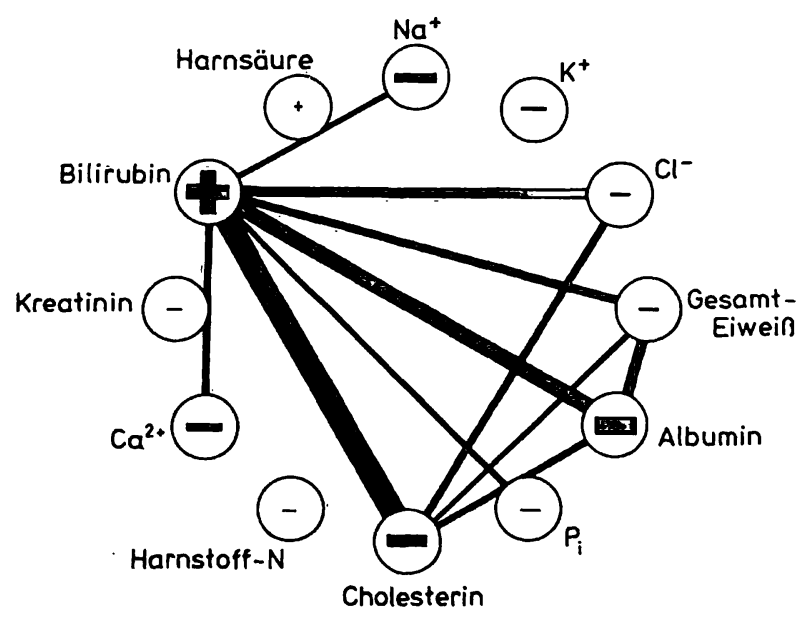

Abb. 10. Krankheitsspezifisches Abweichungsmuster der Patienten mit Lebercirrhosen, bezogen auf das Referenzkollektiv der gesunden Männer. Alle Werte wurden der XTransformation unterzogen. Einzelheiten siehe Text. 
eine starke Zunahme des Bilirubins. Des weiteren fällt eine allgemeine Erniedrigung der Elektrolytkonzentrationen auf. Doch ergeben sich auch Unterschiede zwischen beiden Graphiken. So kommt bei den X-Rängen dem Cholesterin eine zentralere Rolle zu als bei den untransformierten Werten. Umgekehrt ist es bei dem Harnstoff-Stickstoff, bei dem die Abweichung sogar das Vorzeichen wechselt.

Die entsprechenden Abweichungsmuster für die Männer mit Hyperparathyreoidismus zeigen die Abbildungen 11 und 12. Beide Bilder werden dominiert durch eine massive Erhöhung des Calciums. Doch kommt bei den X-transformierten Werten die Abnahme des anorganischen Phosphors stärker zur Geltung. Bei den Frauen ergeben sich ähnliche Bilder.

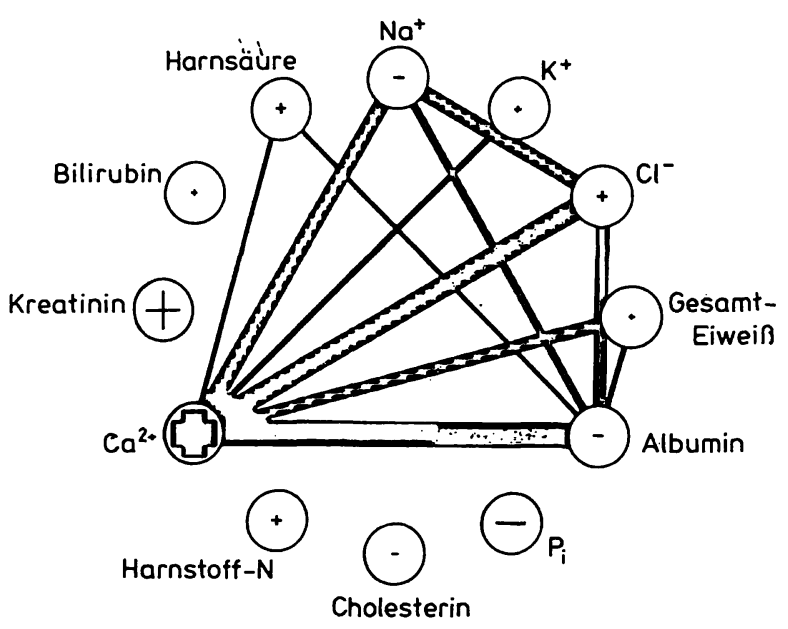

Abb. 11. Krankheitsspezifisches Abweichungsmuster der Patienten mit Hyperparathyreoidismus $(n=34)$, bezogen auf das Referenzkollektiv der gesunden Männer. Die Werte wurden nicht transformiert. Einzelheiten siehe Text.

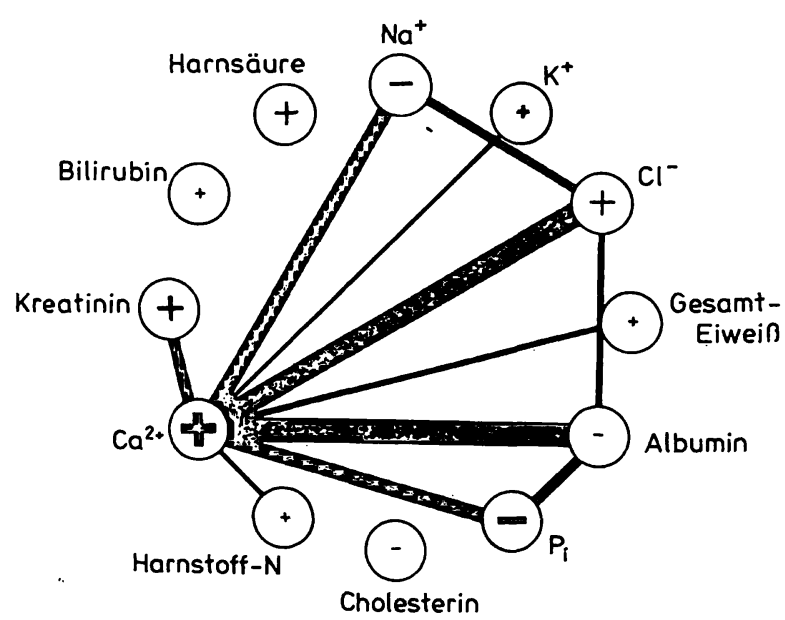

Abb. 12. Krankheitsspezifisches Abweichungsmuster der Patienten mit Hyperparathyreoidismus $(n=34)$, bezogen auf das Referenzkollektiv der gesunden Männer. Alle Werte wurden der X-Transformation unterzogen. Einzelheiten siehe Text.

\section{Diskussion}

Die X-Transformation von van der Waerden wurde von uns in einem heuristischen Ansatz zu dem Zweck durchgeführt, bei den Daten des jeweiligen Referenzkollektivs eine möglichst gute Úbereinstimmung mit multivariat-normalverteilten Größen zu erreichen. Man beachte, daß die X-Transformation hier etwas anders angewandt wurde als von van der Waerden vorgeschlagen. Dort wurde die XTransformation konzipiert, um eine möglichst mächtige verteilungsfreie Alternative für den unverbundenen t-Test zu haben. Der Nullhypothese entsprechend, daß beide Datensätze zu demselben Kollektiv gehören, wird die X-Transformation dort auf beide Datensätze gleichzeitig angewandt. Hier sind dagegen zunächst nur die Werte des Referenzkollektivs von dieser Transformation betroffen, den Werten der hiermit $\mathrm{zu}$ vergleichenden Probanden werden dann X-Ränge zugeordnet, die sich aus dem Vergleich der Probandenwerte mit den Referenzwwerten ergeben. Man könnte zwar auch hier, einer Nullhypothese folgend, die Probandenwerte in das Referenzkollektiv integrieren, um danach die X-Transformation durchzuführen, doch wäre damit ein unnötig großer Rechenaufwand verbunden, der kaum einen Einfluß auf die Prüfgröße $V_{X}^{2}$ hätte. Wir halten daher das hier durchgeführte Verfahren für žweckmäßiger. Für die Durchführung der Rechnungen braucht man dann nur die Referenzwerte mit den zugehörigen $\mathrm{X}$-Werten zu speichern und kann dann die X-Ränge der Probanden durch einfache Vergleiche ermitteln.

Die Ergebnisse der Rechnungen zeigen, da $\beta$ in den zweidimensionalen Randverteilungen durch die XTransformation sehr gute Annäherungen an normalverteilte Größen erreicht werden. Das bedeutet aber natürlich noch nicht, daß hierdurch eine Überführung in eine multivariate Normalverteilung erreicht werden kann. Abgesehen davon, daß der Wertebereich der X-Transformation endlich und diskret ist (was nicht viel ausmacht), kann dies verschiedene Ursachen haben. Unserer Meinung nach sollte man die X-Transformation in den folgenden Fällen nicht durchführen:

1. Das zugrunde liegende Referenzkollektiv zeigt starke Inhomogenitäten. Dies kann vorkommen, wenn das Referenzkollektiv aus Pätienten besteht, die gleichzeitig mehrere Krankheiten haben. In diesem Fall liegt eine Mischverteilung vor, die multivariat multimodal sein kann. Man muß sich daher bemühen, nach Möglichkeit nur "reine Fälle" in das Referenzkollektiv zu übernehmen. Wenn das Datenmaterial groß genug ist, kann mån versuchen, sol- 
che „Klumpenbildungen“ in den Daten mit Hilfe der Clusteranalyse (12) zu erkennen. Es sei jedoch bemerkt, daß eine Inhomogenität in den Daten, die durch die unterschiedlichen Schweregrade der Erkrankung entsteht, nicht schädlich ist, falls die Einwirkung der Erkrankung auf die einzelnen klinischchemischen Kenngrößen durch den Schweregrad monoton gesteuert wird.

2. Der Wertebereich der Größen ist bei dem Referenzkollektiv nicht konvex. Dies könnte vorkommen, wenn die Verteilung gewissermaßen „Löcher“ hätte, wenn also gewisse Gebiete innerhalb des Wertebereiches der Größen ausgespart würden. Ein solches Verhalten ist jedoch schwer vorstellbar und angesichts der großen topologischen Schwierigkeiten in höherdimensionalen Räumen wohl auch kaum zu entdecken. Die zweidimensionalen Randverteilungen geben jedenfalls keinen Hinweis auf solches Verhalten. Nichtkonvexe Gebiete können auch entstehen, wenn der Wertebereich strahlenförmig ist, die Werte also entlang gewisser Vorzugsrichtungen liegen und die dazwischen liegenden Gebiete ausgespart würden. Dies würde sich unter anderem in einer starken Heteroskedastizität in den Daten äuBern. $\mathrm{Zu}$ solchen Heteroskedastizitäten kommt es auch, wenn die Korrelationen zwischen den Größen etwa vom Alter der Personen abhängen, es also sozusagen zu einer Drehung der Referenzellipsoide kommt. Bei dem Auftreten solcher Schwierigkeiten muß man entweder die Altersvariabilität einschränken, d.h. Altersklassen bilden, oder man muß zu wesentlich aufwendigeren ganz verteilungsfreien Verfahren greifen. Die hiẹ angewandte Technik ist ja nur insofern verteilungsfrei, als die Daten vor der Rechnung in Rangzahlen verwandelt wurden. Dies wurde hier "quasi-verteilungsfrei“ genannt. Unsere Daten haben keine Hinweise auf solche Schwierigkeiten gegeben, was indessen zum Teil auf die geringen Stichprobenumfänge zurückgeführt werden kann. Auf jeden Fall stellt die Entdeckung solcher verteilungsmäßigen Besonderheiten in den Daten ein Problem für sich dar.

3. Man möchte die Probandenwerte nur mit ein und demselben Referenzkollektiv vergleichen, zum Beispiel bei indiskriminierten Routineuntersuchungen mit gesunden Referenzpersonen. Hier ist es besser, nach geeigneten, formelmäßig angebbaren Transformationen zu suchen, weil diese mit einem geringeren Informationsverlust verbunden sind als die X-Transformation. Dies äußert sich besonders bei extremen Werten, die aus dem Wertebereich der X-Transformation herausfallen.
Allgemein haben die Rechnungen gezeigt, daß die $X$-Transformation die Tendenz hat, die Werte der Prüfgröße $V^{2}$ zu reduzieren. So ergeben sich beispielsweise in den untransformierten Daten Werte für $V^{2}$ von bis zu über 8000 , wohingegen die entsprechenden $V_{X}^{2}$-Werte nur bis knapp über 100 reichen. Dies liegt zweifellos an der Reduktion der extremen Patientenwerte durch die X-Transformation, bei der alle Werte höchstens auf den Rand des durch die X-Transformation definierten Gitters zu liegen kommen. Interessant und wichtig ist jedoch die Tatsache, daß die Signifikanz der $\mathrm{V}^{2}$-Werte durch die $X$-Transformation nicht berührt wurde. Nur bei einer Patientin mit Hyperparathyreoidismus wurde ein schwach signifikanter Wert in einen Wert dicht unter der Signifikanzschwelle verwandelt.

Die Vorteile der X-Transformation werden erst deutlich, wenn man Patientenkollektive, deren Daten in der Regel schief verteilt sind, als Referenzkollektive benutzen will. Hierbei ergeben sich im einzelnen folgende Vorteile:

1. Die Suche nach geeigneten Transformationen entfällt. Die X-Transformation ist zudem auf Rechenanlagen leicht und schnell durchzuführen. Es genügt durchaus, einfache Approximationen zu benutzen, etwa die bei Abramowitz \& Stegun angegebenen Formeln (13).

2. Es wird eine gute Approximation an eine mehrdimensionale Normalverteilung erreicht, wenn dies überhaupt durch eine Transformation der Randverteilungen möglich ist. Auch wenn dies nicht möglich sein sollte, sind die Ergebnisse vermutlich besser, als wenn man überhaupt nicht transformiert hätte. Es werden auch etwaige nichtlineare Zusammenhänge zwischen den Größen begradigt, soweit sie monoton sind. Außerdem wird der Einfluß von extremen Werten (die etwa durch Ausreißer im Referenzkollektiv entstehen könnten) reduziert.

3. Die Empfindlichkeit der rechnerischen Prüfung wird gesteigert bei schiefverteilten Referenzkollektiven, unter anderem, weil es nicht mehr zu einer Ubberschätzung der Varianzen und einer Unterschätzung der Korrelationen kommt. Vorausgesetzt werden muß hierzu jedoch, daß dạs Referenzkollektiv hinreichend groß ist und daher ein hinreichend feines und umfassendes X-Raster resultiert.

Wie die Rechnungen gezeigt haben, ist eine Trennung zwischen verschiedenen Patientenkollektiven möglich. Ein Blick auf die berechneten krankheitsspezifischen Abweichungsmuster (Abb. 9-12) zeigt, daß auch nach der X-Transformation die charakteri- 
stischen Merkmale erhalten bleiben. Dennoch kommt es zu einer leichten Verschiebung der Akzente. So kommt es bei den Männern mit Hyperparathyreoidismus zu einer Hervorhebung des anorganischen Phosphors bei den transformierten Daten. Man hat den Eindruck, daß nach dem Zurückdrängen der enormen Calciumanhebung bei diesen $\mathrm{Pa}$ tienten die übrigen, noch in den Daten verborgenen Abweichungen von dem normalen Verhalten nach der Transformation besser zur Geltung kommen.

Die Prüfgröße $V_{X}^{2}$ ist nicht nur eine Hilfe bei der diagnostischen Zuordnung von klinisch-chemischen Datensätzen, sondern das hiermit nach dem Konzept vom kollektiven Verhalten berechnete Abweichungsmuster gibt auch in komprimierter Form einen Einblick in die pathobiochemischen Veränderungen bei bestimmten Krankheitsbildern, der auf andere Weise wohl kaum zu erhalten wäre. Dies sei anhand der Abbildungen 10 bis 12 erläutert.

Bei Männern mit Lebercirrhose dominieren als klinisch-chemische Leitsymptome eine ausgeprägte Hyperbilirubinämie, eine starke Erniedrigung des Albumins sowie eine allgemeine Absenkung der Elektrolytkonzentrationen. Die Abnahme des Albumins, dessen Synthese in der Leber deutlich eingeschränkt ist, wird begleitet von einem Anstieg des Bilirubins als Ausdruck vorwiegend prä- und postmikrosomaler Störungen des Bilirubinstoffwechsels und -transports. Das Gesamt-Eiweiß ist ebenfalls erniedrigt, da es das Albumin einschließt.

Das Kenngrößenpaar Bilirubin-Cholesterin erscheint am stärksten im Bild der diagnostischen Wertigkeit ausgeprägt. Die Erniedrigung des Cholesterins im Serum wird allgemein bei Leberparenchymerkrankungen beobachtet und ist auf ein $\mathrm{Ab}$ sinken des Esteranteils bei unverändertem freien Cholesterin zurückzuführen.

Auffällig ist die Erniedrigung sämtlicher untersuchter Elektrolyte. Da bei der Lebercirrhose häufig ein sekundärer Hyperaldosteronismus vorliegt, wäre zunächst eher eine Erhöhung des Natriums und des Chlorids im Serum zu erwarten. Berücksichtigt man aber die Expansion des extrazellulären Natriumund Chloridraumes z. B. beim Vorliegen eines Ascites, so sind die Hyponatriämie und Hypochlorämie trotz Zunahme des Gesamtkörpernatriums nicht überraschend. Außerdem können diese Veränderungen auch durch therapeutische Maßnahmen wie kochsalzsarme Nahrung und Diuretikamedikation verursacht werden. Die niedrigen Kaliumwerte im Serum sind Ausdruck eines echten Kaliummangels als Folge des sekundären Hyperaldosteronismus. Als
Erklärung für die niedrigen Harnstoff-N-Werte kommt eine Einschränkung der Synthese bei normaler Nierenfunktion in Betracht. Zusätzlich spielt auch hier ein Verdünnungseffekt durch Zunahme des Plasmavolumens eine Rolle.

Das Abweichungsmuster bei Männern mit primärem Hyperparathyreoidismus ist ebenfalls mit unseren derzeitigen pathobiochemischen Vorstellungen vereinbar. Klinisch-chemische Leitsymptome sind hier die massive Hypercalcämie und die ebenfalls stark ausgeprägte Hypophosphatämie. Das Kenngrößenpaar: Albumin-Calcium ist in allen maximalen Viererkombinationen vertreten. Die Calciumanhebung als Folge einer Úberproduktion von Parathyrin ist das entscheidende Kennzeichen bei Patienten mit primärem Hyperparathyreoidismus. Sie ist kombiniert mit einer Hypophosphatämie, die durch eine verminderte tubuläre Phosphatrückresorption bedingt ist.

Die im Abweichungsmuster erkennbaren Veränderungen der übrigen Elektrolyte lassen sich auf eine metabolische Acidose zurückführen. Diese wird ausgelöst durch einen hemmienden Effekt des Parathyrins auf die Rückresorption von Hydrogencarbonat in den Nierentubuli, der zu einer vermehrten Hydrogencarbonat- und Natriumausscheidung führt. Konsekutiv kommt es zu einer Substitution des Hydrogencarbonats und des Natriums im Serum durch Chlorid- und Wasserstoffionen und damit zu einer hyperchlorämischen metabolischen Acidose mit begleitender Hyponatriämie. Im Rahmen der metabolischen Acidose werden Wasserstoffionen aus dem extraz̄ellulären Raum ausgetauscht, woraus eine Erhöhung des Kaliums im Serum resultiert.

Die Erhöhung des Harnstoff- $\mathrm{N}$ und des Kreatinins kann als Hinweis auf eine in fortgeschritteneren Stadien auftretende Einschränkung der Nierenfunktion gedeutet werden. Dafür spricht auch die Verminderung des Albumins im Serum, die als Folge einer bei parathyreogenem Nierenschaden relativ frühzeitig auftretenden Albuminurie anzusehen ist. Auch die Erhöhung der Harnsäurekonzentration beim Hyperparathyreoidismus durch eine verminderte renale Exkretion ist nicht unbekannt.

Auf Grund der bisherigen Rechenergebnisse ist zu erwarten, daß die pathobiochemischen Veränderun= gen auch für andere definierte Krankheitsbilder durch typische Abweichungsmuster dargestellt werden können. Somit ermöglicht die multivariate Betrachtungsweise nicht nur die Beurteilung von klinisch-chemischen Profilen einzelner Patienten, sondern vermittelt auch Einblicke in pathobiochemische Veränderungen bei bestimmten Krankheiten. 


\section{Literatur}

1. Winkel, P., Lyngbye, J. \& Jörgensen, K. (1972) Scand. J. Lab. Invest. 30, 339-344.

2. Grams, R. R., Johnson, E. A. \& Benson, E. S. (1972) J. Clin. Pathol. 58, 188-200.

3. Kågedal, B., Sandström, A. \& Tibbling, G. (1978) Clin. Chem. 24, 1744-1750.

4. Rehpenning, W., Harm, K., Domesle, Astrid \& Voigt, K. D. (1979) J. Clin. Chem. Clin. Biochem. 17, 565-573.

5. Harm, K., Rehpenning, W., Domesle, Astrid \& Voigt, K. D. (1979) J. Clin. Chem. Clin. Biochem. 17, 517-522.

6. Rehpenning, W. (1983) Multivariate Datenbeurteilung, Statistische Untersuchungen über krankheitsbedingte Lage- und Strukturveränderungen klinisch-chemischer Kenngrößen. Med. Informatik und Statistik 43, Springer, Berlin, Heidelberg, New York, Tokyo.
7. Hotelling, H. (1931) Ann. Math. Stat. 2, 360-378.

8. Harm, K. (1974) Med. Progr. Technol. 3, 45-55.

9. Technicon (1971) Reagent Data Handbook for the Technicon SMA 12/60 System. Technical Publication No. THO0160-10. Technicon Instruments Corporation, Tarrytown.

10. Van der Waerden, B. L. (1965) Mathematische Statistik. Springer, Berlin-Göttingen-Heidelberg.

11. Victor, N. (1979) Meth. Inform. Med. 17, 120.

12. Jardine, N. \& Sibson, R. (1971) Mathematical Taxanomy. John Wiley \& Sons, London, New York, Sydney, Toronto.

13. Abramowitz, M. \& Stegun, I. A. (1965) Handbook of Mathematical Functions. Dover Publications, New York.

Priv.-Doz. Dr. Wolfgang Rehpenning Institut für Mathematik und Datenverarbeitung in der Medizin

Universitäts-Krankenhaus Hamburg-Eppendorf Martinistraße 52, Pavillon 70

D-2000 Hamburg 20 
$\therefore:$ : 